\title{
Tetraspanin CD81 regulates HSV-1 infection
}

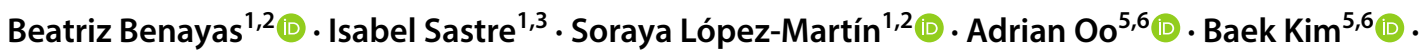 \\ Maria J. Bullido ${ }^{1,3,4}$ (]) Jesús Aldudo ${ }^{1,3,4}$ - María Yáñez-Món ${ }^{1,2,7}$ (])
}

Received: 3 April 2020 / Accepted: 27 May 2020 / Published online: 4 June 2020

(c) Springer-Verlag GmbH Germany, part of Springer Nature 2020

\begin{abstract}
Different members of the tetraspanin superfamily have been described to regulate different virus infectious cycles at several stages: viral entry, viral replication or virion exit or infectivity. In addition, tetraspanin CD81 regulates HIV reverse transcription through its association with the dNTP hydrolase SAMHD1. Here we aimed at analysing the role of CD81 in Herpes simplex virus 1 infectivity using a neuroblastoma cell model. For this purpose, we generated a CD81 KO cell line using the CRISPR/Cas9 technology. Despite being CD81 a plasma membrane protein, CD81 KO cells showed no defects in viral entry nor in the expression of early protein markers. In contrast, glycoprotein B and C, which require viral DNA replication for their expression, were significantly reduced in CD81 KO infected cells. Indeed, HSV-1 DNA replication and the formation of new infectious particles were severely compromised in CD81 KO cells. We could not detect significant changes in SAMHD1 total expression levels, but a relocalization into endosomal structures was observed in CD81 KO cells. In summary, CD81 KO cells showed impaired viral DNA replication and produced greatly diminished viral titers.
\end{abstract}

Keywords Tetraspanin $\cdot$ CD81 $\cdot$ Herpes simplex-1 $\cdot$ SAMHD1 $\cdot$ Viral replication

\section{Introduction}

Tetraspanin-enriched microdomains (TEM) are the result of the interaction between tetraspanins, other membrane proteins and lipids. They play important roles in several physiological and pathological processes related with cell adhesion, migration or membrane functions [1]. It has been

Edited by Luise Florin.

Jesús Aldudo and María Yáñez-Mó contributed equally to this manuscript.

This article is part of the Special Issue on Tetraspanins in Infection and Immunity.

Jesús Aldudo

jaldudo@cbm.csic.es

$\triangle$ María Yáñez-Mó

maria.yannez@uam.es

1 Centro de Biología Molecular, "Severo Ochoa" (C.S.I.C.U.A.M.), Universidad Autónoma de Madrid, C/Nicolás Cabrera 1, 28049 Madrid, Spain

2 Centro de Biología Molecular Severo Ochoa, Instituto de Investigación Sanitaria La Princesa (IIS-IP), 28006 Madrid, Spain described that TEMs can serve as a platform for viral infection. Tetraspanins interact with multiple host membrane factors required in this process, collaborating in viral entry, assembly or egress steps [2-5]. For example, CD9 and CD81 regulate virus-cell membrane fusion in human immunodeficiency virus type I (HIV-1) infection [6]; CD81 facilitates Hepatitis $\mathrm{C}$ virus (HCV) entry in cells [7, 8]; different tetraspanins regulate entry of papilloma and cytomegalovirus [9, 10]; and TEMs are the budding sites for Influenza A virus (IAV) [11] and Herpes simplex virus 1 (HSV-1) [12].

3 Centro de Investigacion Biomédica en Red Sobre Enfermedades Neurodegenerativas (CIBERNED), Madrid, Spain

4 Instituto de Investigación Sanitaria "Hospital la Paz" (IdIPaz), Madrid, Spain

5 Department of Pediatrics, Emory University School of Medicine, Atlanta, GA 30322, USA

6 Center for Drug Discovery, Children's Healthcare of Atlanta, Atlanta, GA 30322, USA

7 Departamento de Bioquímica y Biología Molecular, UAM, Centro de Biología Molecular Severo Ochoa, Lab 412, C/Nicolás Cabrera, 1, 28049 Madrid, Spain 
In addition to events occurring at the plasma membrane, we recently described the functional connection between tetraspanin CD81 and SAMHD1 [13], which regulates HIV reverse transcription. SAMHD1 is a deoxyribonucleotide triphosphate (dNTP) hydrolase that plays an important role in cell homeostasis maintenance, controlling optimal dNTP levels for an accurate DNA synthesis and repair [14]. SAMHD1 has also been described as an effector of innate immunity and innate antiviral response, function that has been widely reviewed [15-18]. SAMHD1 antiviral effect was first described for HIV-1 [19]. In the last years, its antiretroviral activity has been demonstrated in different cell types, such as monocytes or dendritic cells [20-22]. Although SAMHD1 has the capacity to bind and degrade HIV-1 genomic RNA [23-25], it is now accepted that viral restriction by SAMHD1 is mainly mediated by its dNTPase function, given that reverse transcription is tightly dependent on cellular dNTP levels [17, 22, 26]. Our group previously reported that tetraspanin CD81 is implicated on HIV-1 infection by allowing viral reverse transcription to occur through SAMHD1 control [13]. CD81 associates with SAMHD1 to regulate its expression and subcellular localization. In the lack of CD81, a SAMHD1-dependent decrease in the dNTPs intracellular pool is observed, causing deficient reverse transcription.

Similarly to the reverse transcription of HIV-1, SAMHD1 has an impact on other viruses by regulating dNTP levels. This has been already proven for Vaccinia virus [27], Hepatitis B virus [28-30] and Herpes simplex virus type 1 (HSV-1) [27, 31]. Herpes DNA replication blockage by SAMHD1 has been demonstrated in macrophages and non-dividing myeloid cells [27, 31]. However, herpesvirus protein kinases have recently been shown to inhibit by phosphorylation the dNTPase function of SAMHD1 [32, 33].

To address whether CD81 modulates the infection of DNA viruses we chose Herpes simplex virus type 1 (HSV1), a large doubled-stranded DNA virus and member of the Alphaherpesviridae family as a model system. HSV-1 is a neurotropic virus, usually establishing latent infections in the trigeminal ganglia followed by periodic reactivations. Due to these reactivations, HSV-1 may also reach the central nervous system causing acute events like encephalitis and/or establishing lifelong latent infections in the brain. Mounting evidence supports the involvement of neurotropic viruses from the Herpesviridae family, especially HSV-1, in Alzheimer's disease (AD) pathogenesis [34]. This hypothesis argues that latent HSV-1 infection of the central nervous system could be involved in the neurodegenerative process, either via a direct effect of the infectious agent itself or via the associated inflammatory response, or both. It has been reported that reactivation of HSV-1 induces AD-related alterations such as accumulation of amyloid- $\beta$ protein $(A \beta)$, tau hyperphosphorylation and neuroinflammation episodes
[35]. With all this background, we decided to explore the role of CD81 in HSV-1 infection in a neuroblastoma cell model.

\section{Materials and methods}

\section{Cell cultures}

The SK-N-MC human neuroblastoma cell line (HTB10) was purchased from the American Type Culture Collection (ATCC). SK-N-MC cells were grown as monolayers in minimal Eagle's medium (MEM) supplemented with $10 \%$ heat-inactivated foetal calf serum (FCS), $2 \mathrm{mM}$ glutamine and $50 \mu \mathrm{g} / \mathrm{ml}$ gentamicin, at $37^{\circ} \mathrm{C}$ in a $5 \% \mathrm{CO}_{2}$ atmosphere.

CRISPR/Cas9 technology was used for the generation of a CD81 ${ }^{-}$SK-N-MC cell line as described [13]. For CRISPR/ Cas9 plasmids transfection, SK-N-MC cells were washed with PBS and electroporated with $20 \mu \mathrm{g}$ of total DNA in incomplete medium at $200 \mathrm{~V}$ and $975 \mu \mathrm{F}$ (Gene Pulser II, Bio-Rad, Hercules, CA, USA). A second round of transfection was performed 5 days later. One week post-transfection, cells were blocked with $\gamma$-globulin (Sigma) and stained with anti-CD81 5A6 monoclonal antibody. The negative population was isolated using a FACSARIA FUSION Cell Sorter. Cells were routinely tested for CD81 negative expression and used only in the first culture passages.

\section{Antibodies}

Rabbit anti-HSV glycoprotein B and D (gB/gD) antibody was kindly provided by E. Tabares. Antibodies that recognized viral proteins $\mathrm{gC}$ and ICP4 were supplied by Abcam [anti-HSV1 gC Envelope Protein (3G9) and anti-HSV 1 ICP4 Immediate Early Protein [10F1]). Mouse monoclonal anti-tubulin (clone B-5-1-2; T5168), anti-SAMHD1 Abs (SAB1101454 and HPA047072) were obtained from Sigma; and anti-EEA1 610457 from BD Biosciences. Anti-CD81 5A6 monoclonal antibody was kindly donated by S. Levy (Standford).

The secondary antibodies used for immunostaining were horseradish peroxidase-coupled anti-mouse (Vector; PI-2000) and anti-rabbit (Nordic; GAR/IgG(H + L)/PO) antibodies, and species-matching secondary antibodies coupled to 488 and 647 Alexa Fluor fluorochromes (ThermoFisher).

\section{Infection conditions}

The wild-type HSV-1 strain KOS 1.1 was propagated and purified from Vero cells as previously described [36]. SK$\mathrm{N}-\mathrm{MC}$ cells seeded in complete MEM at $70-80 \%$ confluence 
were exposed to HSV-1 at $37^{\circ} \mathrm{C}$ for $1 \mathrm{~h}$. Mock infections were performed using a virus-free suspension. Unbound virus was removed and the cells incubated in complete MEM at $37^{\circ} \mathrm{C}$. Time and multiplicity of infection (moi; expressed as plaque-forming units [pfu] per cell) are indicated in each experiment. The infectious titers of purified virus and cell supernatants were determined by plaque assay [37]. Briefly, the titration of serially diluted HSV-1 and cell supernatant samples was performed in Vero cells grown in 24-well plates. Cells were overlain with a mixture of DMEM containing $2 \% \mathrm{FCS}$ and $0.7 \%$ agar. After $48 \mathrm{~h}$, the cells were fixed and stained overnight with $1 \%$ crystal violet in $5 \%$ formaldehyde and the plaques counted.

\section{Immunofluorescence analysis}

Immunofluorescence assays of viral protein ICP4 were performed on cells grown on coverslips. Samples were fixed in $4 \%$ paraformaldehyde and incubated with the appropriate primary and secondary antibodies. DAPI $(5 \mathrm{mg} / \mathrm{ml})$ was added $10 \mathrm{~min}$ before the end of the procedure to visualize the nuclei. Cells were examined using a Zeiss Axiovert 200 fluorescence microscope. Images were captured by a Spot RT slider digital camera (Diagnostic) using MetaMorphTM imaging software, and processed using Adobe Photoshop CS4.

For confocal microscopy analysis of SAMHD1 subcellular localization, cells were grown on fibronectin-coated coverslips $(20 \mu \mathrm{g} / \mathrm{ml}$; Sigma) and fixed in $4 \%$ PFA. For SAMHD1 staining, blocking and staining solutions were composed of $0.2 \%$ Triton X-100, $\gamma$-globulin and TNB and appropriated primary and secondary antibodies. Samples were washed with TBS-Triton and mounted on coverslips with Flouromount-G (Thermo Fisher) containing DAPI $(0.1 \mu \mathrm{M})$. Confocal images were obtained with an A1R + Nikon confocal microscopy attached to an inverted microscope (Eclipse Ti-E model, Nikon) with a Plan-Apocromatic 60X/1.4 oil immersion objective, using NIS Elements 4.40 acquisition software. Images were analysed with ImageJ.

\section{Flow cytometry}

For extracellular CD81 tetraspanin detection, cells were blocked with $\gamma$-globulin (Sigma) during $20 \mathrm{~min}$ at $4{ }^{\circ} \mathrm{C}$, incubated with primary anti-CD81 5A6 mouse monoclonal antibody for $1 \mathrm{~h}$ at $4{ }^{\circ} \mathrm{C}$ and stained with secondary goat anti-mouse Alexa647 (ThermoFisher) for $30 \mathrm{~min}$ at $4{ }^{\circ} \mathrm{C}$. For intracellular SAMHD1 detection, cells were fixed in $2 \%$ paraformaldehyde and permeabilized in $0.1 \%$ saponin. Cells were blocked $1 \mathrm{~h}$ at room temperature in PBS saponin $0.1 \%, 5 \%$ BSA and $\gamma$-globulin. Primary SAMHD1 rabbit polyclonal antibody and donkey anti-rabbit Alexa647 (ThermoFisher) were incubated in PBS $0.1 \%$ saponin, $0.5 \%$ BSA, $15 \mathrm{mM}$ glycine and $10 \mathrm{mM}$ HEPES solution, at $4^{\circ}$.

Data were acquired with a FACSCantoII flow cytometer and analysed with FlowJo.

\section{Immunoblot analysis}

For immunoblot assays, cells were lysed in RIPA cell lysis buffer $(10 \mathrm{mM}$ Tris-HCl pH 7.5, $50 \mathrm{mM} \mathrm{NaCl}, 0.2 \%$ sodium deoxycholate, $1 \%$ NP-40 and $0.1 \%$ SDS buffer) containing Complete Mini Protease Inhibitor Cocktail (Roche), and incubated for $30 \mathrm{~min}$ at $4{ }^{\circ} \mathrm{C}$. The protein concentration of the lysates was quantified using the BCA Kit (Pierce). Cell lysates were mixed with Laemmli buffer, sonicated, and heated for $5 \mathrm{~min}$ at $100{ }^{\circ} \mathrm{C}$. After electrophoretic separation, the gels were blotted and stained with the appropriate antibodies. The secondary antibodies used for immunostaining were horseradish peroxidase-coupled anti-mouse (Vector; PI-2000) and anti-rabbit (Nordic; GAR/IgG(H + L)/PO) antibodies. Detection by enhanced chemiluminiscence was performed using ECL ${ }^{\mathrm{TM}}$ Western Blotting Detection Reagents (Amersham Biosciences) according to the manufacturer's instructions.

\section{HSV-1 DNA quantification}

The amount of HSV-1 DNA was quantified by real-time quantitative PCR. DNA was extracted using the QIAamp ${ }^{\circledR}$ DNA Mini Kit (QIAGEN). The concentration of viral DNA was then quantified by qPCR with an ABI Prism 7900HT $\mathrm{SD} \circledast$ system (Applied Biosystems) using a custom designed TaqMan probe specific for the US12 viral gene (5'-AGG CGGCCAGAACC- $3^{\prime}$ ). Viral DNA content was then normalized in terms of human genomic DNA, quantified with a predesigned TaqMan probe specific for the $18 S$ gene (Applied Biosystems). The quantification results were represented as viral DNA copy numbers per ng of genomic DNA, as calculated using a standard curve obtained with known quantities of human genomic DNA.

\section{dNTP measurement}

The cellular dNTP amounts were measured as previously described [38]. Briefly, cellular dNTPs were extracted from known numbers of cells, and applied to the HIV-1 RT-based dNTP assay. The determined dNTP amounts were normalized by cell numbers. 


\section{Results}

\section{CD81 gene deletion causes a reduction in HSV-1 late gene expression}

To assess whether CD81 plays a functional role in the infectious cycle of DNA viruses, we chose Herpes Simplex as a model system. Because of the relevance of HSV-1 in the CNS, we generated a CD81-deficient neuroblastoma SK-NMC cell line using the CRISPR/Cas9 technology [13]. CD81 gene deletion was verified by flow cytometry (Fig. 1a) and western blot (Fig. 1b).

Both wt and CD81- SK-N-MC cell lines were infected with HSV-1 at different times and multiplicities of infection (MOI). Efficiency of HSV-1 infection was determined by assessing the levels of expression of viral proteins (Fig. 2). Immunoblotting analyses were performed using antibodies specific for ICP4, an immediate early protein, whose expression begins before HSV-1 DNA replication takes place; glycoprotein B (gB), a $\gamma 1$ "leaky-late" protein, the expression
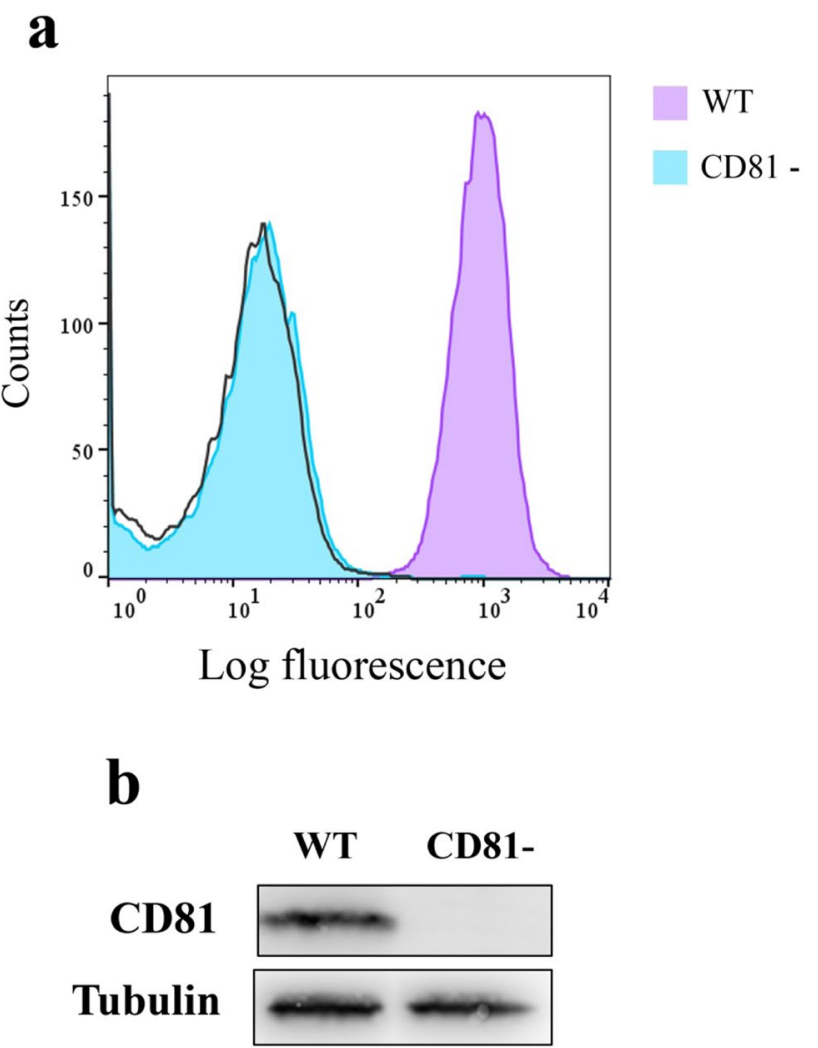

Fig. 1 Characterization of CD81- SK-N-MC cells. a Flow cytometry histograms of CD81 expression in wild-type (violet) and CD81deficient SK-N-MC cells (blue) after staining with 5A6 anti-CD81 $\mathrm{mAb}$. Negative control histogram is shown in the black line profile. $\mathbf{b}$ Western-blot detection of CD81 protein in both cell lines. Tubulin is shown as loading control of which is not strictly dependent on viral DNA synthesis but once DNA replication has commenced its expression is dramatically increased; and glycoprotein $\mathrm{C}(\mathrm{gC})$, which belongs to the class of $\gamma 2$ " true late" genes, so its expression requires viral DNA synthesis. In all conditions, the levels of viral proteins $\mathrm{gB}$ and $\mathrm{gC}$ were clearly decreased in CD81-deficient cells in comparison to wild type ones (Fig. 2a). However, protein levels of ICP4 protein, which is expressed earlier in the viral cycle showed no significant differences between wt and CD81- cells (Fig. 2b).

\section{CD81 gene deletion does not affect HSV-1 entry step}

Viral protein expression profile suggested that CD81 may be affecting a late stage in the viral cycle, but since CD81 is expressed at the plasma membrane and has been reported to be implicated on viral entry of some viruses [7], we directly quantitated HSV-1 entry by assessing the number of cells that were infected and thus expressed nuclear ICP4 protein. ICP4 is expressed at the very early steps of the infectious process and is detected as early as $2 \mathrm{~h}$ post-infection. First, ICP4 shows a diffuse distribution in the nucleus, later accumulating at pre-replicative sites, which appear as small dotted structures. As the infection progresses, large globular replication compartments, termed viral replication compartments, are generated, where ICP4 is involved in the transcription and replication of viral DNA [39].

ICP4-positive nuclei were counted for both wt and $\mathrm{CD} 81^{-}$cells at both 3 and 5 h post-infection, and no differences in the number of infected cells were observed at any time or MOI (Fig. 3a, b). Different staining patterns are apparent in the cultures since cell infection was performed at $37^{\circ} \mathrm{C}$, so that it would not be synchronized in all cells. These data are consistent with the results obtained in the immunoblotting experiments that showed no changes in total ICP4 levels in wt compared to CD81-deleted cells infected with HSV-1 (Fig. 2b), indicating that CD81 does not affect viral entry into human neuroblastoma cells.

\section{CD81 regulates SAMHD1 subcellular localization}

The molecular interaction of CD81 with the dNTP triphosphohydrolase SAMHD1 [13], may be a possible mechanism behind the inhibition of the late stages of HSV-1 infectious cycle that we observed in neuroblastoma cells upon CD81 gene deletion. To address this issue, we analysed both SAMHD1 expression and localization in SK-N-MC cells in presence or absence of CD81 expression. No differences were found in total levels of SAMHD1 by western blot (Fig. 4a) or flow cytometry analyses (data not shown). However, as we found in our previous report in Hela cells [13], SAMHD1 subcellular localization was indeed altered in CD81 knockout neuroblastoma cells, in which we observed 
Fig. 2 CD81 gene deletion reduces HSV-1 protein expression. Wt and CD81-deficient SK-N-MC cells were infected with HSV-1 at the indicated multiplicities of infection and the levels of viral proteins were analysed by western blot at the indicated times post infection (hpi). a Levels of the viral glycoproteins $\mathrm{B}$ and $\mathrm{C}$ ( $\mathrm{gB}$ and $\mathrm{gC}$ ) in cells with 1 and $3 \mathrm{pfu} /$ cell for $18 \mathrm{~h}(18 \mathrm{hpi})$ or with 0.1 and $0.3 \mathrm{pfu} / \mathrm{cell}$ for $42 \mathrm{~h}$ (42 hpi). The blots shown are representative of three independent experiments. A tubulin blot is provided as a control for equal loading. The ratio of viral proteins to tubulin with respect to infected wt cells is shown below the blots. b Levels of ICP4 in wt and CD81-deficient SK-N-MC cells infected with HSV-1 with 1 or 3 pfu/cell during 3 or $5 \mathrm{~h}$ (hpi). A tubulin blot as a control for equal loading is also shown. The ratio of ICP4 to tubulin with respect to infected wt cells is shown below the blots $\mathbf{a}$
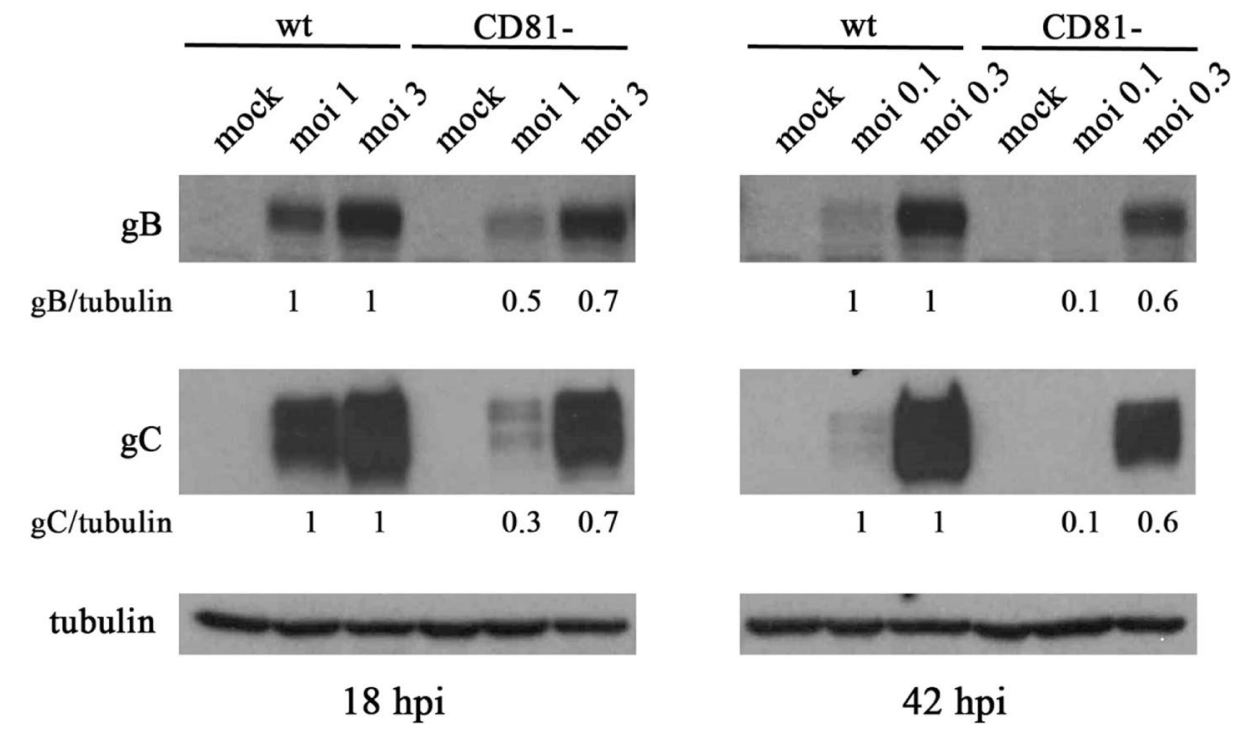

42 hpi

b

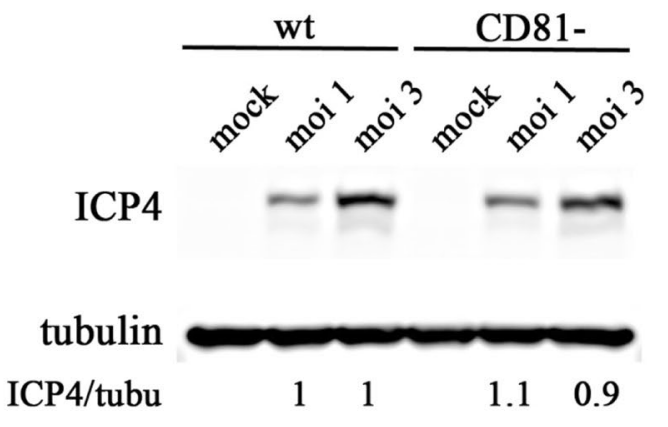

3 hpi

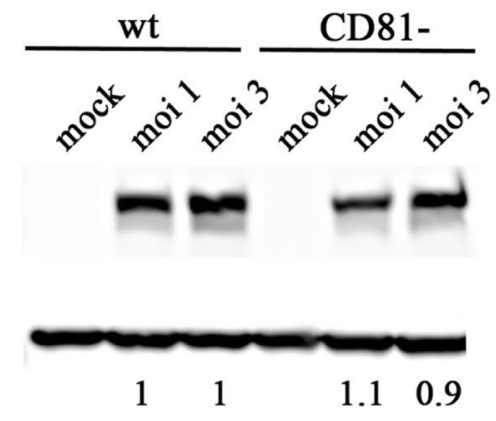

5 hpi an increase in the number of cells showing SAMHD1 endosomal localization (Fig. 4b).

\section{CD81 gene deletion impairs HSV-1 DNA replication and reduces viral titers}

Although we could not detect changes in SAMHD1 total expression levels, viral protein expression data suggested an effect on late stages of viral infection dependant on DNA replication. Thus, we next quantitated viral DNA replication by PCR. qPCR analysis of viral DNA replication showed a significant decrease in viral DNA content in CD81-deleted cells in comparison to wild-type cells (Fig. 5a). Finally, the effect on HSV-1 titres was measured by plaque assays. A strong reduction in the production of infectious HSV-1 particles was seen in CD81-deficient cells, which, in some experiments, produced no infectious particles (Fig. 5b). Viral DNA levels were specially reduced after $42 \mathrm{~h}$ of infection, time at which at least one replication cycle is likely to have taken place.

All these results prove that CD81 positively regulates HSV-1-efficient infection and point to an effect in a late stage in the viral cycle, such as DNA replication.

\section{Discussion}

Several tetraspanins have been described to play important roles in viral infections [2], although most reports focus on the role of tetraspanins and tetraspanin-enriched microdomains in those processes of the viral cycle that occur at the level of the plasma membrane (entry, exit, syncytia formation) [2-5]. We have recently described that the tetraspanin CD81 collaborates in HIV-1 infection by facilitating viral reverse transcription through the control of the protein levels and subcellular localization of the dNTPase SAMHD1 [13]. All viruses depend for their cycle progression on the supply 
$\mathbf{a}$
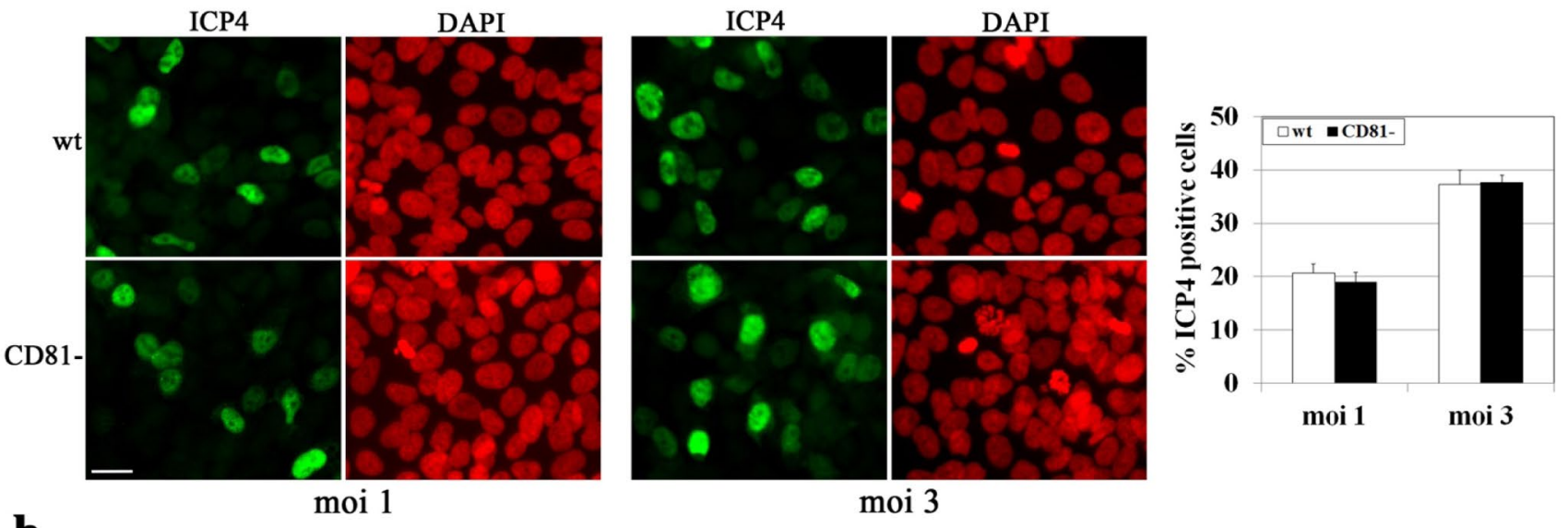

b

moi 1
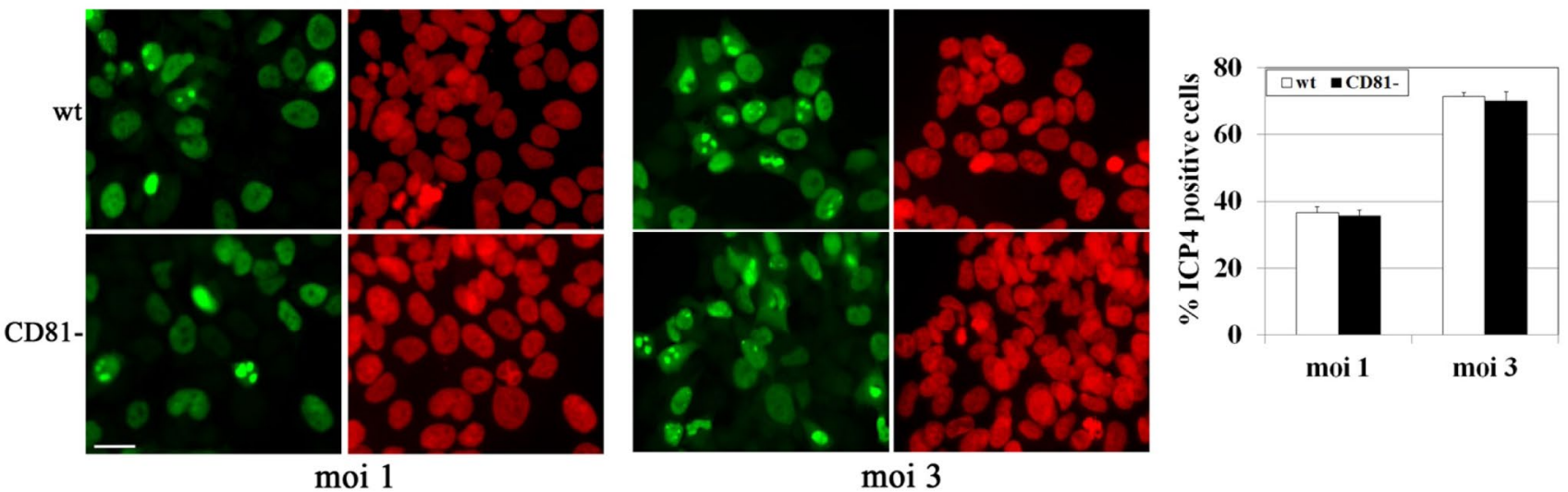

Fig. 3 CD81 gene deletion does not affect HSV-1 entry. Immunofluorescence analysis of wt and CD81-deficient SK-N-MC cells infected with HSV-1 at a moi of 1 and 3 pfu/cell for $3 \mathrm{~h}$ (a) or $5 \mathrm{~h}(\mathbf{b})$. The immunoreactivity of ICP4 viral protein is shown. Nuclei are stained with DAPI. Quantification of infected cells by ICP4 staining was per- formed and the graphs show the percentage of ICP4-positive cells. At least 300 nuclei were counted from 3 independent experiments (minimum of 100 nuclei per experiment), Graphs depict the mean \pm SEM of the 3 experiments. Scale bars: $20 \mu \mathrm{m}$ of nucleotides from the infected cell, so the mechanism described for HIV could be in principle more general. Thus, we aimed to address whether a similar mechanism could occur in other type of virus such as DNA viruses, affecting in this case viral DNA replication. We chose Herpes simplex as a model virus, because of their wide distribution in the population and the relevance it has been pointed out in the etiopathology of some neurodegenerative diseases such as Alzheimer [34].

To directly address the role of CD81 in HSV-1 infection, we generated a KO cell line using the CRISPR/Cas9 technology. Since CD81 is expressed on the plasma membrane, gene-deleted cells were selected by flow cytometry, so that we were not working with clonal populations. Tetraspanins are a relatively large superfamily (33 members in humans) from which a selected repertoire is present in all cell types of the human organism, thus, there is no tetraspanin-null cell in higher eukaryotes. This ubiquitous representation of tetraspanins points out to their fundamental role in membrane structure. Therefore, upon gene deletion, compensatory mechanisms commonly occur. This is evidenced by the very severe phenotype of double KO mice [40] compared to the usually mild- or cell-typespecific effect of single tetraspanin deletion [41-44]. Thus, CD81 KO cells were used only in the first cell passages after selection to minimize these compensatory effects. In those early passages, we could not detect a compensatory upregulation of other tetraspanin members tested (CD9, CD151 and CD82) although we cannot rule out that other members of the family are upregulated since there is a lack of appropriated detection reagents for most of them.

We observed that CD81 gene deletion reduces HSV-1 infection. No differences were observed in viral entry or expression of the immediately early ICP4 protein, while all 
Fig. 4 Regulation of SAMHD1 expression and localization by CD81 expression. a SAMHD1 levels were analysed by western blot in wild-type (WT) and CD81-deficient SK-N-MC $\left(\mathrm{CD} 81^{-}\right)$cells. Tubulin was used as loading control. Representative blots are shown. The plot depicts the ratio of SAMHD1 to tubulin as the mean \pm SEM of six independent experiments. b Confocal microscopy analysis of SAMHD1 subcellular localization after SAMHD1 immunostaining. Nuclei were stained with DAPI. Representative images are shown. (Scale bar: $40 \mu \mathrm{m})$. Graph depicts the percentage of cells (mean \pm SEM) showing SAMHD1 relocalization to endosomal structures from 4 fields of view in 2 independent experiments (a minimum of 200 cells/experiment) $* * p<0.01$ in a Student's t-test

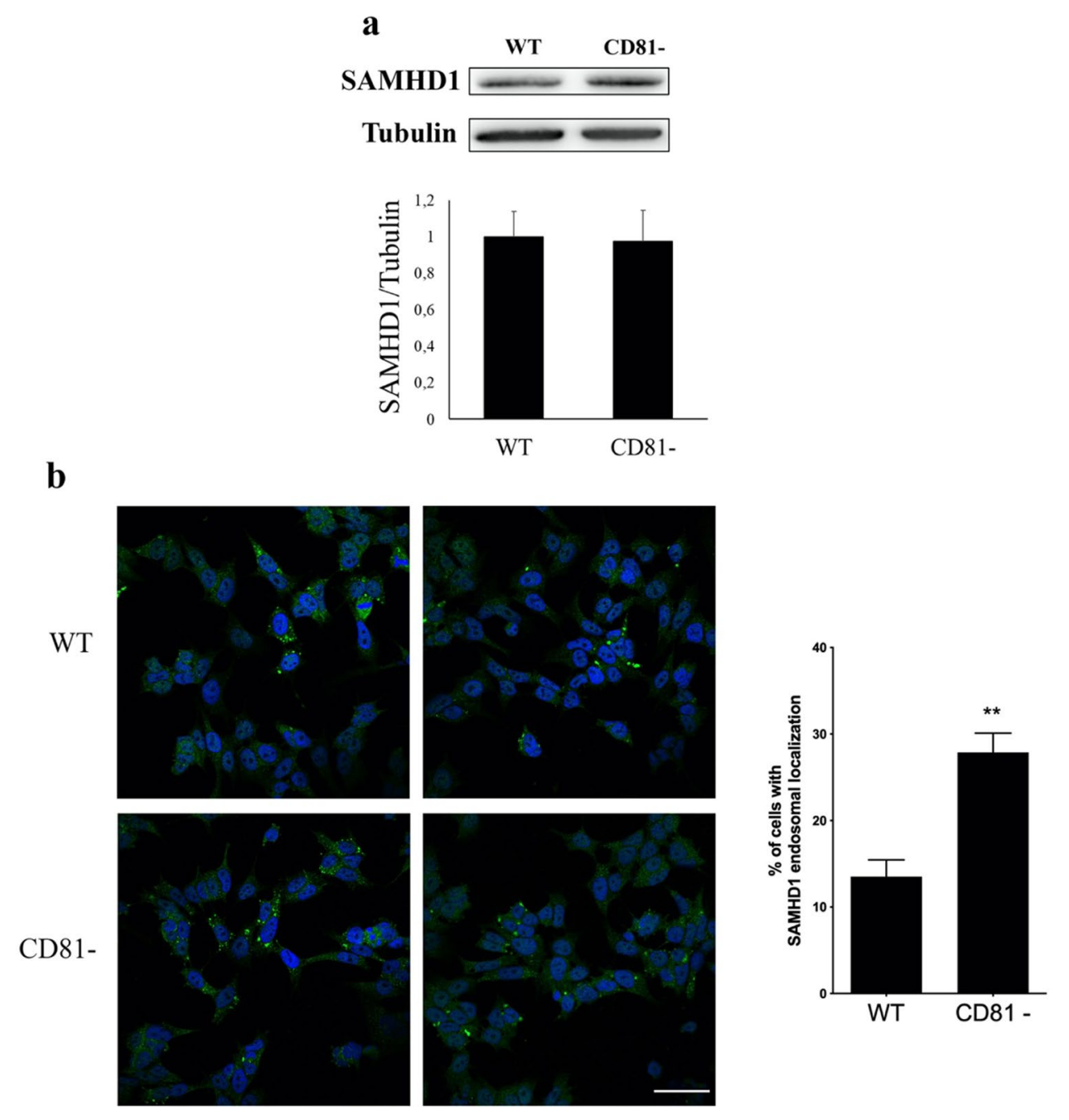

the events that require DNA replication were impaired, such as expression of late proteins ( $\mathrm{gB}$ and $\mathrm{gC}$ ), viral DNA replication itself, or active virion production, suggesting that the role of tetraspanin CD81 may be related to DNA replication.

In our previous report [13], we described that CD81 expression regulated SAMHD1 degradation by the proteasome in a HIV permissive HeLa cell model. Although the enzyme is predominantly localized in the nucleus, it has also been reported its presence in the cytoplasm [13, 45, 46], where dNTPs synthesis takes place. Shuttling from the nucleus to the cytoplasm seems to be regulated by its oxidation state [46]. SAMHD1 was shown to relocalized to the plasma membrane upon CD81 crosslinking, while in the absence of the tetraspanin, cytoplasmic SAMHD1 accumulated in endosomal structures and its proteasomal degradation was significantly reduced. The increment in SAMHD1 expression levels was accompanied by a reduction in the cytosolic concentration of dNTPs in the cell, thus hampering the HIV reverse transcription step. Here, we have observed a similar relocalization of SAMHD1 to endosomal structures in neuroblastoma cells upon CD81 gene deletion. However, neither SAMHD1 total expression levels nor cytosolic dNTP levels (not shown) were altered in CD81-deficient neuroblastoma cells. These discrepancies could be caused by the different cell lines employed, that could have different SAMHD1 turnover in basal conditions. Lately, viral kinases conserved in the Herpesviridae family, have been reported to inhibit SAMHD1 function by phosphorylation [32, 33]. In our experiments, SAMHD1 expression and localization and cellular dNTP levels were only analysed in non-infected cells. The change in SAMHD1 subcellular location as well as the effect of CD81 gene deletion on viral replication may suggest that upon viral infection, SAMHD1 activity may be affected. SAMHD1 is regulated by either oxidation [47] or phosphorylation [32, 33, 48]. The fact that different posttranslational modifications occur on SAMHD1 may also difficult a precise quantitation of its protein expression levels, since these modified forms may be differentially detected by specific antibodies against the enzyme. 
Fig. 5 CD81 gene deletion reduces HSV-1 DNA replication and viral titers. a Quantification of viral DNA by real-time quantitative PCR of six independent experiments performed in triplicate. The data represent the mean \pm SEM of the six experiments performed expressed as a percentage with respect to infected wt cells (one sample $t$ test; ${ }^{*} p<0.05$; $* * p<0.01 ; * * * p<0.001)$. b Viral titers were determined by plaque assays in the culture medium of infected SK-N-MC cells. Graph data represent the mean \pm SEM of at least three experiments and are expressed as a percentage with respect to wt cells (one sample $t$ test; $* p<0.05 ; * * * p<0.001)$ a
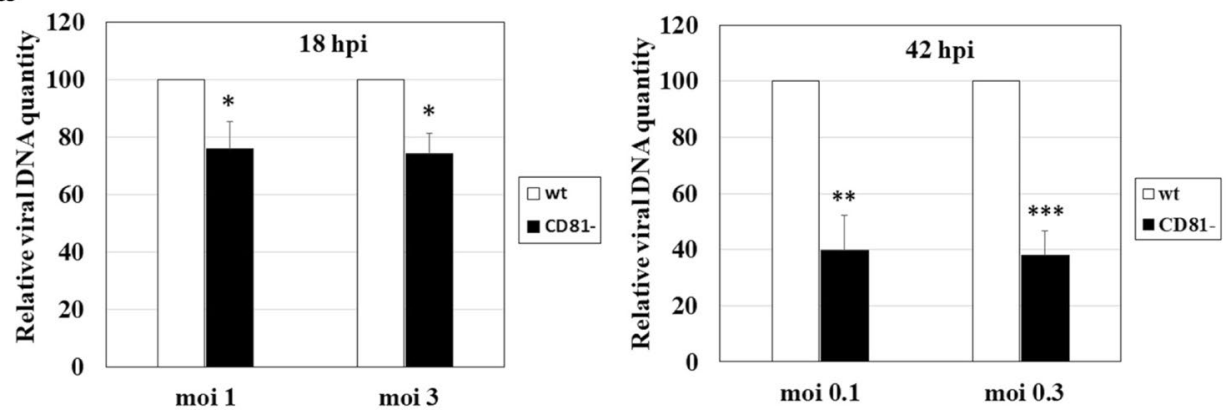

b

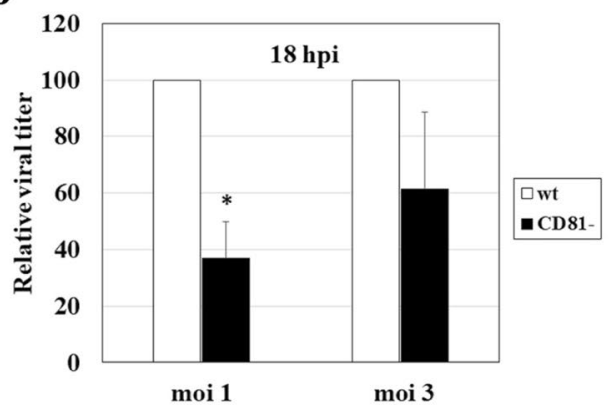

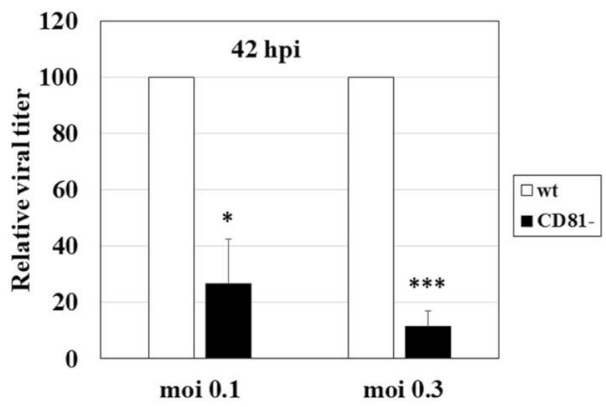

Some DNA viruses such as HSV-1 present enzymes involved in dNTPs synthesis to ensure enough levels for viral replication [49]. Cytoplasmic SAMHD1 may be responsible of the degradation of newly synthetized dNTPs, which could explain why CD81-deficient cells show especially lower infection levels at longer times. Cellular basal dNTPs pool may be enough for the first round of replication, but subsequent replication cycles would require newly synthetized dNTPs, which can be degraded by SAMHD1 in the cytosol. It would be thus interesting in the future to analyse dNTP levels in the course of an infection in wild-type and CD81-deficient cells.

In summary, our data demonstrate that CD81 gene deletion impairs HSV-1 infection by affecting DNA replication step. Because of the reiterative appearance of emerging viral infections such as the pandemic caused by SARS-CoV-2 virus, it is of crucial relevance to explore the role of cellular targets, like tetraspanins, that may be involved in the viral cycle of a wide variety of these infectious agents.

Aknowledgements We want to thank Victor Toribio for critical reading of the manuscript.

Funding This work has been supported by grants BIO2017-86500-R from Ministerio Español de Economía y Competitividad (MINECO) to MY-M and SAF2017-85747-R to MJB as well as USA NIH R01 AI136581 and AI150451 to BK. The institutional grants of Fundación Ramón Areces and Banco de Santander to the Centro de Biología Molecular Severo Ochoa are gratefully acknowledged. BB was supported by a predoctoral contract from Garantía Juvenil CAM program.

\section{Compliance with ethical standards}

Conflict of interest The authors declare no conflict of interest.

\section{References}

1. Yanez-Mo M, Barreiro O, Gordon-Alonso M, Sala-Valdes M, Sanchez-Madrid F (2009) Tetraspanin-enriched microdomains: a functional unit in cell plasma membranes. Trends Cell Biol 19(9):434-446. https://doi.org/10.1016/j.tcb.2009.06.004

2. Florin L, Lang T (2018) Tetraspanin assemblies in virus infection. Front Immunol 9:1140. https://doi.org/10.3389/fimmu .2018 .01140

3. Hantak MP, Qing E, Earnest JT, Gallagher T (2019) Tetraspanins: architects of viral entry and exit platforms. J Virol. https ://doi.org/10.1128/JVI.01429-17

4. Martin F, Roth DM, Jans DA, Pouton CW, Partridge LJ, Monk PN, Moseley GW (2005) Tetraspanins in viral infections: a fundamental role in viral biology? J Virol 79(17):10839-10851. https://doi.org/10.1128/JVI.79.17.10839-10851.2005

5. Suarez H, Rocha-Perugini V, Alvarez S, Yanez-Mo M (2018) Tetraspanins, another piece in the HIV-1 replication puzzle. Front Immunol 9:1811. https://doi.org/10.3389/fimmu .2018 .01811

6. Gordon-Alonso M, Yanez-Mo M, Barreiro O, Alvarez S, MunozFernandez MA, Valenzuela-Fernandez A, Sanchez-Madrid F (2006) Tetraspanins CD9 and CD81 modulate HIV-1-induced membrane fusion. J Immunol 177(8):5129-5137. https://doi. org/10.4049/jimmunol.177.8.5129

7. Zhang J, Randall G, Higginbottom A, Monk P, Rice CM, McKeating JA (2004) CD81 is required for hepatitis C virus glycoproteinmediated viral infection. J Virol 78(3):1448-1455. https://doi. org/10.1128/jvi.78.3.1448-1455.2004 
8. Alberione MP, Moeller R, Kirui J, Ginkel C, Doepke M, Stroh LJ, Machtens JP, Pietschmann T, Gerold G (2020) Single-nucleotide variants in human CD81 influence hepatitis $C$ virus infection of hepatoma cells. Med Microbiol Immunol. https://doi.org/10.1007/ s00430-020-00675-1

9. Fast LA, Mikulicic S, Fritzen A, Schwickert J, Boukhallouk F, Hochdorfer D, Sinzger C, Suarez H, Monk PN, Yanez-Mo M, Lieber D, Florin L (2018) Inhibition of tetraspanin functions impairs human papillomavirus and cytomegalovirus infections. Int J Mol Sci. https://doi.org/10.3390/ijms19103007

10. Mikulicic S, Fritzen A, Scheffer K, Strunk J, Cabanas C, Sperrhacke M, Reiss K, Florin L (2020) Tetraspanin CD9 affects HPV16 infection by modulating ADAM17 activity and the ERK signalling pathway. Med Microbiol Immunol. https://doi. org/10.1007/s00430-020-00671-5

11. He J, Sun E, Bujny MV, Kim D, Davidson MW, Zhuang X (2013) Dual function of CD81 in influenza virus uncoating and budding. PLoS Pathog 9(10):e1003701. https://doi.org/10.1371/journ al.ppat. 1003701

12. Wang L, Liu L, Che Y, Wang L, Jiang L, Dong C, Zhang Y, Li Q (2010) Egress of HSV-1 capsid requires the interaction of VP26 and a cellular tetraspanin membrane protein. Virol J 7:156. https ://doi.org/10.1186/1743-422X-7-156

13. Rocha-Perugini V, Suarez H, Alvarez S, Lopez-Martin S, Lenzi GM, Vences-Catalan F, Levy S, Kim B, Munoz-Fernandez MA, Sanchez-Madrid F, Yanez-Mo M (2017) CD81 association with SAMHD1 enhances HIV-1 reverse transcription by increasing dNTP levels. Nat Microbiol 2(11):1513-1522. https://doi. org/10.1038/s41564-017-0019-0

14. Franzolin E, Pontarin G, Rampazzo C, Miazzi C, Ferraro P, Palumbo E, Reichard P, Bianchi V (2013) The deoxynucleotide triphosphohydrolase SAMHD1 is a major regulator of DNA precursor pools in mammalian cells. Proc Natl Acad Sci USA 110(35):14272-14277. https://doi.org/10.1073/pnas.1312033110

15. Antonucci JM, St Gelais C, Wu L (2017) The dynamic interplay between HIV-1, SAMHD1, and the innate antiviral response. Front Immunol 8:1541. https://doi.org/10.3389/fimmu 2017.01541

16. Li M, Zhang D, Zhu M, Shen Y, Wei W, Ying S, Korner H, Li J (2017) Roles of SAMHD1 in antiviral defense, autoimmunity and cancer. Rev Med Virol. https://doi.org/10.1002/rmv.1931

17. Majer C, Schussler JM, Konig R (2019) Intertwined: SAMHD1 cellular functions, restriction, and viral evasion strategies. Med Microbiol Immunol 208(3-4):513-529. https://doi.org/10.1007/ s00430-019-00593-x

18. Mauney CH, Hollis T (2018) SAMHD1: Recurring roles in cell cycle, viral restriction, cancer, and innate immunity. Autoimmunity 51(3):96-110. https://doi.org/10.1080/08916934.2018.14549 12

19. Lahouassa H, Daddacha W, Hofmann H, Ayinde D, Logue EC, Dragin L, Bloch N, Maudet C, Bertrand M, Gramberg T, Pancino G, Priet S, Canard B, Laguette N, Benkirane M, Transy C, Landau NR, Kim B, Margottin-Goguet F (2012) SAMHD1 restricts the replication of human immunodeficiency virus type 1 by depleting the intracellular pool of deoxynucleoside triphosphates. Nat Immunol 13(3):223-228. https://doi.org/10.1038/ni.2236

20. Behrendt R, Schumann T, Gerbaulet A, Nguyen LA, Schubert N, Alexopoulou D, Berka U, Lienenklaus S, Peschke K, Gibbert K, Wittmann S, Lindemann D, Weiss S, Dahl A, Naumann R, Dittmer U, Kim B, Mueller W, Gramberg T, Roers A (2013) Mouse SAMHD1 has antiretroviral activity and suppresses a spontaneous cell-intrinsic antiviral response. Cell Rep 4(4):689-696. https:// doi.org/10.1016/j.celrep.2013.07.037

21. Gramberg T, Kahle T, Bloch N, Wittmann S, Mullers E, Daddacha W, Hofmann H, Kim B, Lindemann D, Landau NR (2013)
Restriction of diverse retroviruses by SAMHD1. Retrovirology 10:26. https://doi.org/10.1186/1742-4690-10-26

22. White TE, Brandariz-Nunez A, Valle-Casuso JC, Amie S, Nguyen L, Kim B, Brojatsch J, Diaz-Griffero F (2013) Contribution of SAM and HD domains to retroviral restriction mediated by human SAMHD1. Virology 436(1):81-90. https://doi.org/10.1016/j.virol .2012.10.029

23. Beloglazova N, Flick R, Tchigvintsev A, Brown G, Popovic A, Nocek B, Yakunin AF (2013) Nuclease activity of the human SAMHD1 protein implicated in the Aicardi-Goutieres syndrome and HIV-1 restriction. J Biol Chem 288(12):8101-8110. https:// doi.org/10.1074/jbc.M112.431148

24. Choi J, Ryoo J, Oh C, Hwang S, Ahn K (2015) SAMHD1 specifically restricts retroviruses through its RNase activity. Retrovirology 12:46. https://doi.org/10.1186/s12977-015-0174-4

25. Ryoo J, Choi J, Oh C, Kim S, Seo M, Kim SY, Seo D, Kim J, White TE, Brandariz-Nunez A, Diaz-Griffero F, Yun CH, Hollenbaugh JA, Kim B, Baek D, Ahn K (2014) The ribonuclease activity of SAMHD1 is required for HIV-1 restriction. Nat Med 20(8):936-941. https://doi.org/10.1038/nm.3626

26. Amie SM, Noble E, Kim B (2013) Intracellular nucleotide levels and the control of retroviral infections. Virology 436(2):247254. https://doi.org/10.1016/j.virol.2012.11.010

27. Hollenbaugh JA, Gee P, Baker J, Daly MB, Amie SM, Tate J, Kasai N, Kanemura Y, Kim DH, Ward BM, Koyanagi Y, Kim B (2013) Host factor SAMHD1 restricts DNA viruses in nondividing myeloid cells. PLoS Pathog 9(6):e1003481. https://doi. org/10.1371/journal.ppat.1003481

28. Chen Z, Zhu M, Pan X, Zhu Y, Yan H, Jiang T, Shen Y, Dong X, Zheng N, Lu J, Ying S, Shen Y (2014) Inhibition of Hepatitis B virus replication by SAMHD1. Biochem Biophys Res Commun 450(4):1462-1468. https://doi.org/10.1016/j.bbrc.2014.07.023

29. Jeong GU, Park IH, Ahn K, Ahn BY (2016) Inhibition of hepatitis B virus replication by a dNTPase-dependent function of the host restriction factor SAMHD1. Virology 495:71-78. https:// doi.org/10.1016/j.virol.2016.05.001

30. Sommer AF, Riviere L, Qu B, Schott K, Riess M, Ni Y, Shepard C, Schnellbacher E, Finkernagel M, Himmelsbach K, Welzel K, Kettern N, Donnerhak C, Munk C, Flory E, Liese J, Kim B, Urban S, Konig R (2016) Restrictive influence of SAMHD1 on Hepatitis B Virus life cycle. Sci Rep 6:26616. https://doi. org/10.1038/srep26616

31. Kim ET, White TE, Brandariz-Nunez A, Diaz-Griffero F, Weitzman MD (2013) SAMHD1 restricts herpes simplex virus 1 in macrophages by limiting DNA replication. J Virol 87(23):12949-12956. https://doi.org/10.1128/JVI.02291-13

32. Deutschmann J, Schneider A, Gruska I, Vetter B, Thomas D, Kiessling M, Wittmann S, Herrmann A, Schindler M, Milbradt J, Ferreiros N, Winkler TH, Wiebusch L, Gramberg T (2019) A viral kinase counteracts in vivo restriction of murine cytomegalovirus by SAMHD1. Nat Microbiol 4(12):2273-2284. https ://doi.org/10.1038/s41564-019-0529-z

33. Zhang K, Lv DW, Li R (2019) Conserved herpesvirus protein kinases target samhd 1 to facilitate virus replication. Cell Rep 28(2):449-459. https://doi.org/10.1016/j.celrep.2019.04.020

34. Itzhaki RF (2017) Herpes simplex virus type 1 and Alzheimer's disease: possible mechanisms and signposts. FASEB J 31(8):3216-3226. https://doi.org/10.1096/fj.201700360

35. De Chiara G, Piacentini R, Fabiani M, Mastrodonato A, Marcocci ME, Limongi D, Napoletani G, Protto V, Coluccio P, Celestino I, Li Puma DD, Grassi C, Palamara AT (2019) Recurrent herpes simplex virus-1 infection induces hallmarks of neurodegeneration and cognitive deficits in mice. PLoS Pathog 15(3):e1007617. https://doi.org/10.1371/journal.ppat.1007617

36. Burgos JS, Ramirez C, Sastre I, Bullido MJ, Valdivieso F (2002) Involvement of apolipoprotein $E$ in the hematogenous 
route of herpes simplex virus type 1 to the central nervous system. J Virol 76(23):12394-12398. https://doi.org/10.1128/ jvi.76.23.12394-12398.2002

37. Santana S, Recuero M, Bullido MJ, Valdivieso F, Aldudo J (2012) Herpes simplex virus type I induces the accumulation of intracellular beta-amyloid in autophagic compartments and the inhibition of the non-amyloidogenic pathway in human neuroblastoma cells. Neurobiol Aging 33(2):430-433. https://doi. org/10.1016/j.neurobiolaging.2010.12.010

38. Diamond TL, Roshal M, Jamburuthugoda VK, Reynolds HM, Merriam AR, Lee KY, Balakrishnan M, Bambara RA, Planelles V, Dewhurst S, Kim B (2004) Macrophage tropism of HIV-1 depends on efficient cellular dNTP utilization by reverse transcriptase. J Biol Chem 279(49):51545-51553. https://doi. org/10.1074/jbc.M408573200

39. de Bruyn KA, Uprichard SL, Chen M, Knipe DM (1998) Comparison of the intranuclear distributions of herpes simplex virus proteins involved in various viral functions. Virology 252(1):162-178. https://doi.org/10.1006/viro.1998.9450

40. Jin Y, Takeda Y, Kondo Y, Tripathi LP, Kang S, Takeshita H, Kuhara H, Maeda Y, Higashiguchi M, Miyake K, Morimura O, Koba T, Hayama Y, Koyama S, Nakanishi K, Iwasaki T, Tetsumoto S, Tsujino K, Kuroyama M, Iwahori K, Hirata H, Takimoto T, Suzuki M, Nagatomo I, Sugimoto K, Fujii Y, Kida H, Mizuguchi K, Ito M, Kijima T, Rakugi H, Mekada E, Tachibana I, Kumanogoh A (2018) Double deletion of tetraspanins CD9 and CD81 in mice leads to a syndrome resembling accelerated aging. Sci Rep 8(1):5145. https://doi.org/10.1038/s41598-018-23338-X

41. Deng J, Dekruyff RH, Freeman GJ, Umetsu DT, Levy S (2002) Critical role of CD81 in cognate T-B cell interactions leading to Th2 responses. Int Immunol 14(5):513-523. https://doi. org/10.1093/intimm/14.5.513

42. Kelic S, Levy S, Suarez C, Weinstein DE (2001) CD81 regulates neuron-induced astrocyte cell-cycle exit. Mol Cell Neurosci 17(3):551-560. https://doi.org/10.1006/mcne.2000.0955

43. Le Naour F, Rubinstein E, Jasmin C, Prenant M, Boucheix C (2000) Severely reduced female fertility in CD9-deficient mice.
Science 287(5451):319-321. https://doi.org/10.1126/scien ce.287.5451.319

44. Miyado K, Yamada G, Yamada S, Hasuwa H, Nakamura Y, Ryu F, Suzuki K, Kosai K, Inoue K, Ogura A, Okabe M, Mekada E (2000) Requirement of CD9 on the egg plasma membrane for fertilization. Science 287(5451):321-324. https://doi.org/10.1126/ science.287.5451.321

45. Baldauf HM, Pan X, Erikson E, Schmidt S, Daddacha W, Burggraf M, Schenkova K, Ambiel I, Wabnitz G, Gramberg T, Panitz S, Flory E, Landau NR, Sertel S, Rutsch F, Lasitschka F, Kim B, Konig R, Fackler OT, Keppler OT (2012) SAMHD1 restricts HIV-1 infection in resting CD4(+) T cells. Nat Med 18(11):16821687. https://doi.org/10.1038/nm.2964

46. Mauney CH, Rogers LC, Harris RS, Daniel LW, Devarie-Baez NO, Wu H, Furdui CM, Poole LB, Perrino FW, Hollis T (2017) The SAMHD1 dNTP triphosphohydrolase is controlled by a redox switch. Antioxid Redox Signal 27(16):1317-1331. https://doi. org/10.1089/ars.2016.6888

47. Patra KK, Bhattacharya A, Bhattacharya S (2019) Molecular dynamics investigation of a redox switch in the anti-HIV protein SAMHD1. Proteins 87(9):748-759. https://doi.org/10.1002/ prot.25701

48. Hu J, Qiao M, Chen Y, Tang H, Zhang W, Tang D, Pi S, Dai J, Tang N, Huang A, Hu Y (2018) Cyclin E2-CDK2 mediates SAMHD1 phosphorylation to abrogate its restriction of HBV replication in hepatoma cells. FEBS Lett 592(11):1893-1904. https ://doi.org/10.1002/1873-3468.13105

49. Weller SK, Coen DM (2012) Herpes simplex viruses: mechanisms of DNA replication. Cold Spring Harb Perspect Biol 4(9):a013011. https://doi.org/10.1101/cshperspect.a013011

Publisher's Note Springer Nature remains neutral with regard to jurisdictional claims in published maps and institutional affiliations. 